Artículo

\title{
Protocolo de análisis de viabilidad de semillas de chía mediante test de tetrazolio
}

\author{
María Johana González Vera ${ }^{1 \S}$ \\ Tiago Zanatta Aumonde ${ }^{2}$ \\ Geri Eduardo Meneghello² \\ Andréa Bicca Noguez Martins ${ }^{1}$ \\ Yesmina Lezcano Aquino ${ }^{1}$ \\ Pamela Peña ${ }^{2}$ \\ ${ }^{1}$ Posgrado en Ciencia y Tecnología de Semillas-Universidad Federal de Pelotas (UFPel). ${ }^{2}$ Facultad de \\ Ciencias Agrarias-Universidad Nacional de Asunción. \\ ${ }^{\S}$ Autor para correspondencia: joha_ser09@hotmail.com.
}

\section{Resumen}

La chía (Salvia hispanica L.) es una planta herbácea anual, perteneciente a la familia Lamiaceae, se destaca por ser el recurso natural de origen vegetal con mayor contenido de ácidos grasos conocidos hasta ahora. Para obtener éxito en la producción de semillas es necesario utilizar lotes de alta calidad, la cual puede ser evaluada, a través del vigor de estas, en la actualidad una de las principales exigencias para la evaluación del vigor se refiere a la obtención de resultados confiables en un período de tiempo relativamente corto. La prueba de tetrazolio se destaca por ser rápida y confiable. Sin embargo, la metodología para el género Salvia no es referenciada dentro de las normas de la International Seed Testing Association (ISTA), considerando estos hechos, se vuelve importante a la realización del experimento para el desarrollo de un protocolo que permita realizar este análisis. La investigación se realizó en el laboratorio de semillas de la Universidad Feneral de Pelotas, se utilizaron seis lotes de semillas negras de chía y se probaron cuatro concentraciones de sal de tetrazolio $(0.075 \%, 0.1 \%, 0.5 \%$ y $1 \%)$ para evaluar la viabilidad de las semillas. El delineamiento experimental fue en bloques al azar, sometidos al análisis de varianza a través del test $\mathrm{F}$ y posteriormente las medias comparadas entre sí por el test de Tukey, al 5\% de probabilidad, para comparación de las medias. La prueba de tetrazolio conducida en la concentración de $0.075 \%$ es eficiente para evaluar la viabilidad de las semillas de $S$. hispanica L., así como para diferenciar lotes con calidad fisiológica distinta.

Palabras clave: Salvia hispanica L., concentraciones, ISTA, sal de tetrazolio, vigor.

Recibido: agosto de 2019

Aceptado: octubre de 2019 


\section{Introducción}

Salvia hispanica L., es una planta herbácea anual, perteneciente a la familia Lamiaceae, nativa de la región que se extiende desde el sur de México hasta el norte de Guatemala (Chicco et al., 2009). En el período precolombino, era uno de los alimentos básicos utilizados por las civilizaciones que habitaban América Central, siendo superada en importancia sólo por el maíz y los frijoles, pero con mayor destaque que otros cultivos importantes (Ayerza y Coates, 2004).

Estas semillas, además de ser utilizadas como alimento por los indios durante muchos siglos, se ofrecía a los dioses en las ceremonias religiosas para los aztecas. Esta costumbre desapareció hace 500 años, tras la conquista del territorio por los españoles (Ayerza y Coates, 2005). Sin embargo, Jiménez (2010) afirma que la chía sobrevivió en las zonas montañosas de México y Guatemala.

Además de la importancia histórica que posee, es una excelente fuente de minerales, rica en proteínas, lípidos, energía y fibras, el contenido de estos nutrientes en la semilla de esta especie es mayor que de otros cereales, como arroz, cebada, avena, trigo y maíz (Coates y Ayerza, 1996). Se destaca por ser el recurso natural de origen vegetal con mayor contenido de ácidos grasos conocidos hasta ahora, entre ellos el ácido alfa linolénico (omega 3) y ácido linoleico (omega 6).

Los frutos, equivocadamente llamados 'semillas', son esquizocarpo de 1-1.2 mm de ancho y 2-2.2 $\mathrm{mm}$ de longitud, se caracterizan por la mezcla de diferentes tonalidades de colores variando del negro al gris, con manchas irregulares, enrojecidas y marrones e incluso algunas blancas, siendo desconocidas las razones para la presencia de colores diferentes. Cuando empapadas en agua las semillas dan lugar a un líquido gelatinoso debido a la presencia de mucilago en su superficie (Ayerza y Coates, 2004 y 2005).

Actualmente se cultivan en numerosos países como Australia, México, Argentina, Ecuador, Bolivia, Perú, Paraguay y otros (Busilacchi et al., 2013). En Brasil, las regiones del oeste Paranaense y noroeste de Río Grande do Sul comenzaron a invertir en el cultivo de chía en las últimas cosechas, presentando buenos resultados, a pesar de la falta de información acerca de las exigencias nutricionales de la planta (Migliavacca et al., 2014).

Así como las demás especies anuales cultivadas, para obtener éxito en una labranza comercial es necesario utilizar semillas de alta calidad, sin embargo, aún no hay estandarización metodológica para evaluar la viabilidad y la calidad fisiológica para la especie. En la actualidad, una de las principales exigencias para la evaluación del vigor en semillas se refiere a la obtención de resultados confiables en un período de tiempo relativamente corto, permitiendo que el productor pueda tomar decisiones de forma más rápida durante todo el proceso productivo.

Entre los diversos procedimientos utilizados para esta finalidad se destaca la prueba de tetrazolio, el cual se caracteriza por ser una evaluación bioquímica, basada en la actividad de las enzimas deshidrogenasas que catalizan las reacciones respiratorias, presentes en las mitocondrias, localizadas en el interior de las células vegetales (França-Neto et al., 1998). Durante la respiración celular, hay liberación de iones hidrógeno, que reaccionan con la sal de tetrazolio (incoloro y difusible), formando una sustancia de color rojo e insoluble, denominada formazam, delimitando los tejidos vivos de la semilla. 
La coloración resultante de la reacción de la solución de tetrazolio con los iones de hidrógeno es un indicativo de la viabilidad de los tejidos, que ocurre por medio de la detección de la respiración de las células vegetales (AOSA, 1983; França-Neto et al., 1998). La evaluación de la calidad fisiológica a través de la prueba de tetrazolio ha sido difundida para algunas especies y usada en programas de control de calidad de semillas (Costa et al., 2007). Conforme a Deswal y Chand (1997), la prueba de tetrazolio se destaca en este contexto, por ser una técnica que además de evaluar la viabilidad de las semillas, también puede estimar su vigor.

Para algunas especies, como la soja, la técnica es muy estudiada y está bien calibrada, siendo un procedimiento de rutina en muchos laboratorios, sirviendo inclusive como herramienta para la toma de decisión relacionada a la cosecha (França-Neto, 1998). Sin embargo, la metodología para el género Salvia no se hace referencia dentro de las normas del International Seed Testing Association (ISTA) (ISTA, 2003), ni en las reglas para análisis de simientes (RAS) (Brasil, 2009). Considerando estos hechos, se vuelve importante para la realización del experimento para el desarrollo de un protocolo que permita realizar este análisis. Por lo tanto, el objetivo de este trabajo fue evaluar metodología para permitan evaluar la viabilidad en semillas de Salvia hispanica L. utilizando la prueba de tetrazolio.

\section{Materiales y métodos}

El trabajo fue realizado en el período de julio a diciembre, en el Laboratorio de Análisis de Semillas del Departamento de Fitotecnia-Programa de Posgraduación en Ciencia y Tecnología de Semillas de la Universidad Federal de Pelotas. Para el trabajo se utilizaron seis lotes de semillas negras de chía (S. hispanica).

Las semillas fueron sometidas a la prueba de germinación, para lo cual se utilizaron 200 semillas por tratamiento, divididas en cuatro submuestras de 50 semillas, colocadas en cajas tipo gerbox, utilizando como sustrato dos hojas de papel mataborron humedecidas con agua destilada (2.5 veces el peso del papel seco). Posteriormente fueron colocadas en la cámara de germinación (germinador o BOD) a temperaturas alternas de $20-30{ }^{\circ} \mathrm{C}$, según recomendación de las reglas de análisis de semillas (Brasil, 2009) para otras especies del mismo género.

Las evaluaciones de las semillas germinadas se realizaron a los cuatro y siete días. La prueba de primer conteo de germinación fue realizada en forma conjunta con la prueba de germinación y evaluada el cuarto día después de la siembra, los resultados fueron expresados en porcentaje de plántulas normales. Para evaluar la viabilidad de las semillas se utilizaron cuatro concentraciones de cloruro de $2,3,5$-trifeniltetrazolio $(0.075 \%, 0.1 \%, 0.5 \%$ y $1 \%)$, y fueron sometidos al siguiente procedimiento metodológico.

\section{Preparación de la solución}

Inicialmente se preparó una solución patrón al 1\%, mezclando $10 \mathrm{~g}$ de la sal de tetrazolio en 1 litro de agua destilada. Y para las soluciones de trabajo, se realizaron diluciones hasta obtener la concentración deseada. Estas soluciones se almacenaron en frascos de vidrio de color ámbar, en un lugar oscuro y fresco. 


\section{Pre-acondicionamiento}

Fueron inmersas en agua 220 semillas por tratamiento y mantenidas en estas condiciones por un período de 8 horas, a la temperatura de $20{ }^{\circ} \mathrm{C}$, temperatura recomendada para especie del género Ocimum spp., perteneciente a la misma familia botánica de la Salvia, Lamiaceae (Brasil, 2009). Después de ese tiempo fueron colocadas para secar sobre una hoja de papel a temperatura ambiente por un período de una hora o hasta desaparecer el mucilago formado durante la imbibición, el cual dificulta el corte.

\section{Coloración}

Después del pre-acondicionamiento, las semillas fueron seccionadas longitudinalmente a través del centro del eje embrionario con la ayuda de un bisturí y colocadas en vasos, totalmente sumergidos en diferentes concentraciones de sal de tetrazolio $(0.075 \%, 0.1 \%, 0.5 \%$ y $1 \%)$ mantenidas en estas condiciones por $2 \mathrm{~h} \mathrm{a} 30{ }^{\circ} \mathrm{C}$, temperatura recomendada para la especie Ocimum spp. (Lamiaceae) (Brasil, 2009).

\section{Lavado de la muestra}

Después de ser alcanzada la coloración roja las semillas fueron retiradas del ambiente y lavadas con agua y mantenidas sumergidas hasta el momento de la evaluación.

\section{Interpretación}

Se utilizó estereoscopio (10x) para la interpretación. Se evaluaron cuatro submuestras de 50 semillas para cada concentración y se consideraron las siguientes clasificaciones: rojo carmín: tejido vivo y vigoroso y blanco lechoso: tejido muerto.

\section{Procedimientos estadísticos}

El delineamiento experimental fue completamente al azar, para la clasificación de los lotes en cuanto a la germinación los datos fueron sometidos a la transformación ArcSen (raíz x/100) y posteriormente a las medias comparadas entre sí por la prueba de Tukey al 5\% de probabilidad. Para la evaluación de las concentraciones de sal, se consideró un experimento factorial 6 x 4 (seis lotes $\mathrm{x}$ cuatro concentraciones de sal). El procedimiento estadístico fue idéntico al anteriormente descrito, con el diferencial de observar la significancia de la interacción entre los factores y la realización de los respectivos desdoblamientos.

Las siguientes imágenes muestran los pasos necesarios para la realización de la prueba de tetrazolio, cuyas etapas principales son: pre-humedecimiento, secado de mucilago, corte, coloración y evaluación de la viabilidad de las semillas (Figura 1). 


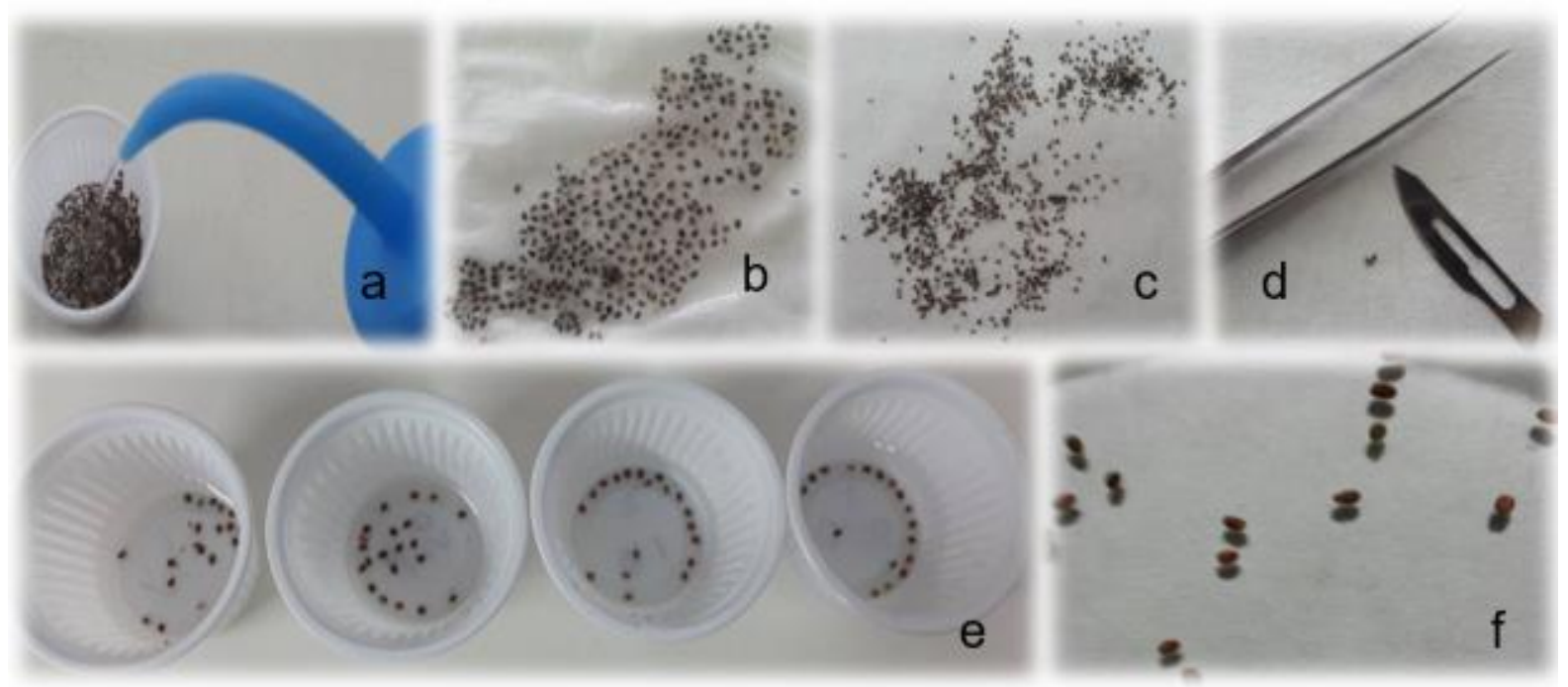

Figura 1. a) pré-humedecimento; b) secado de semillas a temperatura ambiente; c) semillas libres de mucilago; d) corte longitudinal de semillas; e) y f) semillas después de la coloración.

\section{Resultados y discusión}

La coloración de las semillas con sal de tetrazolio $0.075 \%$ y $0.1 \%$ permitieron evaluar sin dificultad la viabilidad, se logró observar que el embrión presenta una coloración más fuerte y resaltante con relación al resto de la semilla; sin embargo, en la concentración de $0.5 \%$ la coloración fue más acentuada, pero aún la lectura fue realizada sin dificultad. Cuando se empleó la solución de 1\%, se observó que la elevada concentración dificultó la interpretación de la viabilidad y causó una coloración excesiva. Probablemente esto ocurrió por el hecho de que esta concentración influenció el proceso de coloración de las semillas, ya que los tejidos del embrión en lugar de coloridos de rosado brillante presentaron una tonalidad roja intensa (similar al color de los tejidos lesionados).

Los patrones de coloración encontrados se presentan en la Figura 2, donde es posible observar variaciones en la tonalidad desde el blanco*semillas inviables (Figura 2g), pasando por las diferentes tonalidades que van de rosa brillante, superficial, uniforme y sin lesiones del embrión hasta coloración roja intensa y profundo (Figura 2 h-l).

Por lo tanto, se verificaron que todas las diluciones presentaron rendimiento en cuanto a la solución de tetrazolio utilizada; sin embargo, el mayor destaque es para la utilización de $0.075 \%$ de sal de tetrazolio. En los tejidos muertos, donde no hay actividad respiratoria, las enzimas deshidrogenasas están inactivas; por lo tanto, no ocurre la reacción con la solución de tetrazolio y consecuentemente, las semillas permanecen descoloridas. Para semillas en proceso de deterioro el desarrollo de la coloración es más rápido, generando un tono rojo más intenso y profundo, mientras que las semillas vigorosas tienen aspecto brillante con coloración rosada a roja (Filho et al., 1987; França-Neto et al., 1998). 


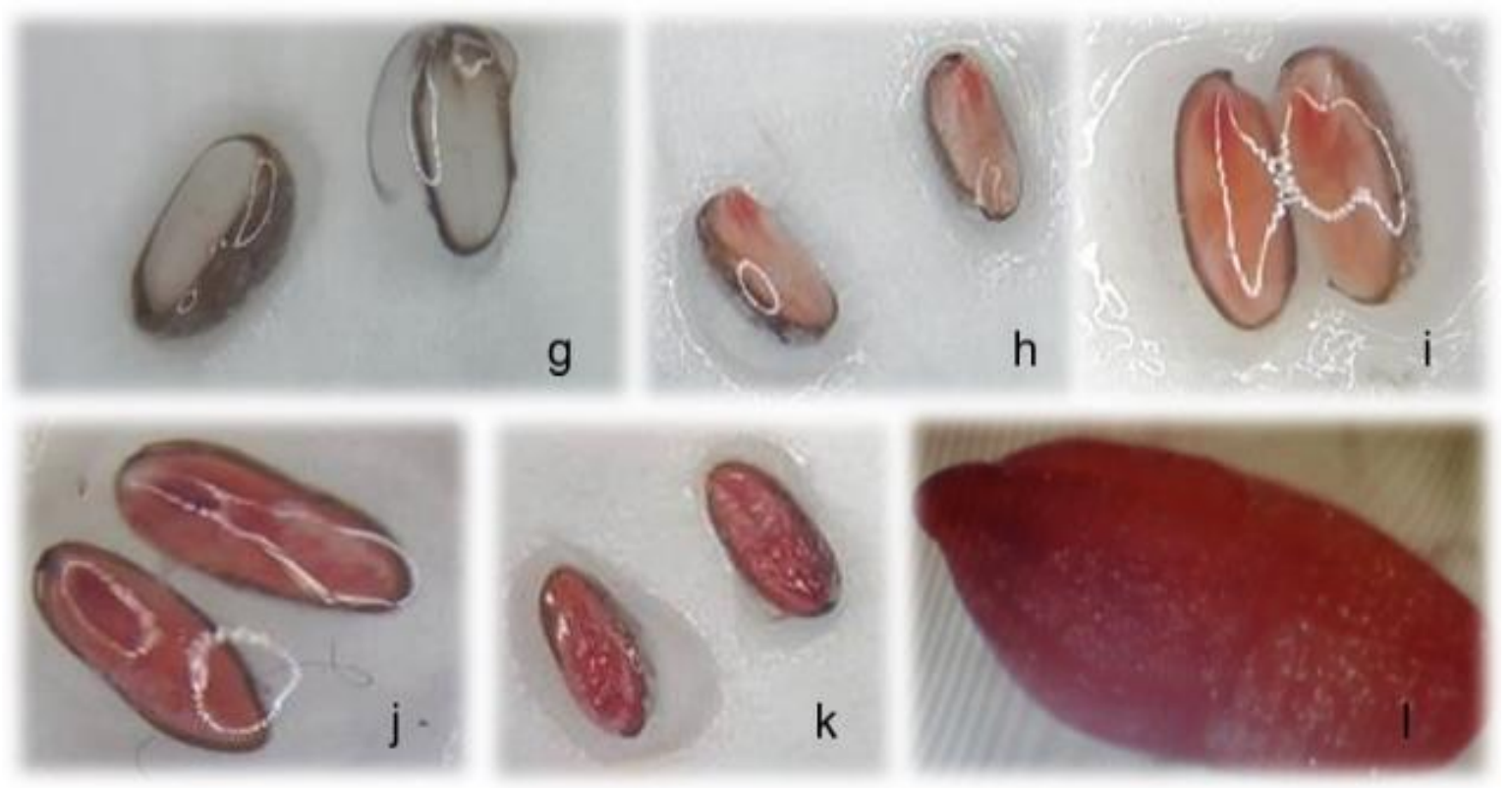

Figura 2. g) semillas no coloreadas, no viables; h) semillas viables cloración $0.075 \%$; i) semillas viables concentración $0.1 \%$; j) semillas viables, concentración $0.5 \%$; k) y l) semillas con coloración roja intensa, concentración $1 \%$ de sal de tetrazolio.

Se destaca que en estudios de esta naturaleza es fundamental la utilización de lotes con calidad diferenciada, pues una determinada metodología debe ser suficientemente sensible y a la vez robusta para detectar diferencias sutiles entre los lotes tanto de alta y de baja calidad.

De acuerdo con la evaluación de la calidad fisiológica de las semillas, realizada por la prueba de germinación tomando en consideración los resultados de la prueba de tetrazolio en las distintas concentraciones de sal (Cuadro 1), los lotes utilizados pueden ser separados en diferentes niveles de calidad, denominados de alta (lotes 2 y 3), media (lote 5) y baja viabilidad (lotes 1,4 y 6 ).

Cuadro 1. Porcentaje de germinación de semillas viables sometidas a pruebas de germinación y viabilidad, utilizando diferentes concentraciones de sal de tetrazolio. FAEM/UFPel.

\begin{tabular}{ccccccc}
\hline \multirow{2}{*}{ Lotes } & \multirow{2}{*}{ Germinación $(\%)$} & PC $(\%)$ & \multicolumn{4}{c}{ Viabilidad en diferentes concentraciones (\%) } \\
\cline { 3 - 6 } & & $0.075 \%$ & $0.1 \%$ & $0.5 \%$ & $1 \%$ \\
\hline L1 & $67 \mathrm{C}$ & $52 \mathrm{C}$ & $76 \mathrm{C} \mathrm{a}$ & $78 \mathrm{C} \mathrm{a}$ & $66 \mathrm{E} \mathrm{b}$ & $76 \mathrm{C} \mathrm{a}$ \\
L2 & $94 \mathrm{~A}$ & $85 \mathrm{~A}$ & $98 \mathrm{~A} \mathrm{a}$ & $96 \mathrm{~A} \mathrm{a}$ & $96 \mathrm{~A} \mathrm{a}$ & $94 \mathrm{~A} \mathrm{a}$ \\
L3 & $95 \mathrm{~A}$ & $87 \mathrm{~A}$ & $96 \mathrm{~A} \mathrm{a}$ & $94 \mathrm{~A} \mathrm{a}$ & $88 \mathrm{~B} \mathrm{~b}$ & $92 \mathrm{~A} \mathrm{ab}$ \\
L4 & $67 \mathrm{C}$ & $59 \mathrm{~B}$ & $70 \mathrm{D} \mathrm{a}$ & $66 \mathrm{D} \mathrm{ab}$ & $68 \mathrm{E} \mathrm{ab}$ & $64 \mathrm{E} \mathrm{b}$ \\
L5 & $79 \mathrm{~B}$ & $43 \mathrm{D}$ & $90 \mathrm{~B} \mathrm{a}$ & $84 \mathrm{~B} \mathrm{~b}$ & $82 \mathrm{C} \mathrm{b}$ & $84 \mathrm{~B} \mathrm{~b}$ \\
L6 & $68 \mathrm{C}$ & $36 \mathrm{D}$ & $74 \mathrm{CD} \mathrm{ab}$ & $76 \mathrm{C} \mathrm{a}$ & $74 \mathrm{D} \mathrm{ab}$ & $70 \mathrm{D} \mathrm{b}$ \\
CV $(\%)$ & 3.75 & 4.48 & \multicolumn{5}{c}{2.61} \\
\hline
\end{tabular}

Medias seguidas por la misma letra mayúscula en la columna y minúscula en la línea, difieren entre sí por el test de Tukey $(p<0.05)$. 
Se observó que, con el uso de la solución a $0.075 \%$ los resultados fueron similares con aquellos obtenidos por la prueba de germinación (Cuadro 1). La utilización de solución de tetrazolio a $0.075 \%$ se ha empleado en el análisis de la viabilidad en semillas de varias especies, como la soja (França-Neto et al., 1998) o maíz (Días y Barros, 1995), calabacita (Barros et al., 2005) y sandia (Bhering et al., 2005), permitiendo el análisis de la viabilidad de las semillas de esas especies con gran rendimiento de solución de tetrazolio durante las evaluaciones.

Para las evaluaciones realizadas con solución de tetrazolio a $0.1 \%$ y $0.5 \%$ (Cuadro 1 ), los resultados también fueron similares a los obtenidos en la prueba de germinación. Sin embargo, cabe resaltar que la comprobada eficiencia, del uso de soluciones de tetrazolio más diluidas se convierte en opciones más adecuadas por permitir el mayor rendimiento de la sal de tetrazolio durante los análisis. Se observaron resultados contrarios en estudios con semillas de caléndula, donde la concentración de $0.5 \%$ de solución de sal de tetrazolio fue muy baja, no siendo posible correlacionar con las demás coloraciones.

Cuando se utilizó el tratamiento con $1 \%$ de concentración de la sal la coloración fue adecuada para el embrión, indicativo de viabilidad. Posiblemente la constitución química de las especies sea muy distinta entre sí, lo que remite a la necesidad de utilizar concentraciones de la sal también diferenciadas. Craviotto et al. (2011) confirmaron que el método con $0.5 \%$ de tetrazolio a $30{ }^{\circ} \mathrm{C}$ fue considerado eficiente para la coloración de soja. Los datos presentados en el Cuadro 2 demuestran que hay significancia para la correlación lineal positiva entre germinación y primer conteo de la germinación en todas las concentraciones de sal estudiadas.

Cuadro 2. Coeficientes de correlación simple de Pearson entre PCG y G con las diferentes concentraciones salinas estudiadas.

\begin{tabular}{ccc}
\hline Concentraciones $(\%)$ & Primer conteo $(\%)$ & Germinación $(\%)$ \\
\hline 0.075 & $0.67^{\text {** }}$ & $0.95^{\text {** }}$ \\
0.1 & $0.66^{\text {** }}$ & $0.92^{* *}$ \\
0.5 & $0.67^{\text {** }}$ & $0.94^{* *}$ \\
1 & $0.66^{\text {** }}$ & $0.91^{* *}$ \\
\hline
\end{tabular}

${ }^{* *}=$ significativo a $1 \%$ de probabilidad por el test $\mathrm{T}$.

Sin embargo, a pesar de ser significativo, el coeficiente de correlación es sustancialmente superior en la variable germinación en comparación con el primer conteo, demostrando eficiencia en la determinación de la viabilidad, ya para pruebas de vigor y es necesaria la calibración de la metodología. Según Filho (2005), la elección de metodología adecuada para el empleo de la prueba de tetrazolio en semillas se basa en la facilidad para diferenciación de tejidos viables e inviables en el estudio económico y en la capacidad de diferenciar lotes de calidad fisiológica distinta.

De esta forma, la concentración de $0.075 \%$ atiende los requisitos de economía y se presta para diferenciación de lotes con relación a la viabilidad y vigor. De acurdo con Santos et al. (2006), la importancia de la prueba de tetrazolio, como instrumento de evaluación de la viabilidad de semillas, se debe a la rapidez en la obtención de sus resultados que pueden ser útiles en áreas de comercialización, beneficiamiento, almacenamiento y producción, sin que esto signifique el compromiso de la prueba de germinación que funciona como una prueba de referencia. 


\section{Conclusiones}

La prueba de tetrazolio utilizando la concentración de $0.075 \%$ a una temperatura de $30{ }^{\circ} \mathrm{C}$ por $2 \mathrm{~h}$ en estufa es eficiente para evaluar la viabilidad de semillas de Salvia hispanica L., así como para diferenciar lotes con calidad fisiológica distinta.

\section{Literatura citada}

AOSA. 1983. Association of Official Seed Analysts. Seed vigor testing handbook. AOSA. Wageningen. $88 \mathrm{p}$.

Ayerza, R. y Coates, W. 2004. Composition of chía (Salvia hispanica) grown in six tropical and subtropical ecosystems of South America. Nova Jérsei. Tropical Science. 3(44):131-135.

Ayerza, R. y Coates, W. 2005. Chia. Rediscovering a forgotten crop of the Aztecs $1^{\text {st }}$ (Ed.). The University of Arizona Press, Tucson, USA. 197 p.

Barros, D. I.; Dias, D. C. F. S.; Bhering, M. C.; Dias, L. A. S. e Araújo, E. F. 2005. Uso do teste de tetrazólio para avaliação da qualidade fisiológica de sementes de abobrinha. Ver. Bras. Sementes. 2(27):165-171.

Bhering, M. C.; Dias, D. C. F. S. e Barros, D. I. 2005. Adequação da metodologia do teste de tetrazólio para avaliação da qualidade fisiológica de sementes de melancia. Ver. Bras. Sementes. 1(27): 176-182.

Busilacchi, H.; Quiroga, M.; Bueno, M.; Di Sapio, O.; Flores, V.; Severin, C. 2013. Evaluación de Salvia hispanica L. cultivada en el sur de Santa Fe (República Argentina). San José de las Lajas. Cultivos Tropicales. 4(34):55-59.

Chicco, A. G.; D’Alessandro, M. E.; Hein, G. J.; Oliva, M. E. and Lombardo, Y. B. 2009. Dietary chia seed (Salvia hispanica L.) rich in alpha-linolenica cidimprovesa diposity and normalizes hypertriacyl glycerolaemia and insulinresistance in dyslipaemicrats. Santa $\mathrm{Fe}$, Argentina British Journal of Nutrition. 1(101):41-50.

Coates, W. and Ayerza, R. 1996. Production potential of chia in northwestern Argentina. Amsterdam. Industrial Cropsand Products. 3(5):229-233.

Costa, N. P.; França Neto, J. B.; Krzyzanowski, F. C. e Henning, A. A. 2007. Metodologia alternativa para o teste de tetrazólio em semente de soja, Londrina. EMBRAPA CNPSo. Circular técnica $39.8 \mathrm{p}$.

Craviotto, R. M.; Arango-Perearnau, M. R. y Gallo, C. 2011. Novedades de la prueba de viabilidad por tetrazolio en soja. Manual de evaluación para semilla de soja. Argentina. Instituto Nacional de Tecnología Agrícola (INTA). 9 p.

Deswal, D. P. and Chand, U. 1997. Standardization of the tetrazolium test for viability estimation in ricebean (Vigna umbellata (Thunb.) Ohwi\&ohashi) seeds. Seed Sci. Technol. 3(25):409-417.

Dias, M. C. L. L. e Barros, A. S. R. 1995. Avaliação da qualidade de sementes de milho. Londrina. IAPAR. Circular 88. 42 p.

França-Neto, J. B.; Krzyzanowski, F. C. e Costa, N. P. 1998. O teste de tetrazólio em sementes de soja. Londrina. EMBRAPA-CNPSo 116. 72 p.

ISTA. 2003. International Seed Testing Association. Bassersdorf, CH-Switzerland. International Rules for Seed Testing. Rules ISTA. 984(1):7-22.

Jiménez, F. E. 2010. Caracterización de compuestos fenólicos presente en la semilla y aceite de chía (Salvia hispanica L.), mediante electroforesis capilar. México. 101 p. 
Marcos Filho, J. 2005. Fisiologia de sementes de plantas cultivadas. Piracicaba. FEALQ. 495 p.

Marcos Filho, J.; Cícero, S. M. e Silva, W. R. 1987. Avaliação da qualidade das sementes. Piracicaba. FEALQ. 230 p.

Migliavacca, R. A.; Vasconcelos, A. L. S.; Santos, C. L. e Baptistella, J. L. C. 2014. Uso da cultura da chia como opção de rotação no sistema de plantio direto. Brasília. Embrapa. 118 p.

Ministério da Agricultura, Pecuária e Abastecimento. Secretaria de Defesa Agropecuária. 2009. Regras para análise de sementes. Ministério da Agricultura, Pecuária e Abastecimento. Secretaria de Defesa Agropecuária. Brasília. MAPA/ACS. 399 p.

Santos, S. R. G.; Paula, R. C.; Fogaça, C. A.; Môro, F. V. e Costa, R. S. 2006. Viabilidade de sementes de Sebastiania commersoniana (Baill.) Smith e Downs (branquilho)Euphorbiaceae-pelo teste de tetrazólio. Científica. 1(34):39-45. 